\title{
Gradient extremals and valley floor bifurcations on potential energy surfaces
}

\author{
Wolfgang Quapp \\ Department of Mathematics, Karl Marx University, DDR-7010, Leipzig, \\ German Democratic Republic
}

(Received March 21, revised August 23/Accepted October 5, 1988)

\begin{abstract}
Gradient extremals are curves in configuration space defined by the condition that the gradient of the potential energy is an eigenvector of the Hessian matrix. Solutions of a corresponding equation go along a valley floor or along a crest of a ridge, if the norm of the gradient is a minimum, and along a cirque or a cliff or a flank of one of the two if the gradient norm is a maximum. Properties of gradient extremals are discussed for simple 2D model surfaces including the problem of valley bifurcations.
\end{abstract}

Key words: Potential energy surfaces - Reaction paths - Saddle points Bifurcation problems - $\mathrm{HCN}$

\section{Introduction}

In the adiabatic approach, the reactive rearrangement of a chemical system is described by a motion of the system point on the potential energy surfaces (PES) from one minimum over a saddlepoint (SP) to another minimum. Once the stationary points have been determined, the next issue of interest is the characterization of possible reaction channels by valleys of the PES and hence, the definition of PES valleys by a formula of their valley floor. This is done in [1] by gradient extremals (GE) (cf also former papers cited there).

The next question is the problem of valley bifurcations. In 1984 we published a paper dealing with the steepest descent path [2], and a further aspect of this matter was discussed in a recent note [3]. A steepest descent does not, however, indicate a bifurcation [2], so here we tackle this problem by tracing the valleys of the PES. We continue the treatment of [1] and use an example of Hoffman, 
Nord and Ruedenberg (HNR) [1] with changed parametrisation. We restrict ourselves to a Euclidean metric and to the two-dimensional (2D) case. Since it contains nearly all phenomena of PES paths of interest arising in real chemical systems and is already complicated. The $N$-dimensional problem has been outlined in [1], and a numerical procedure which traces a nonbifurcated $N$ dimensional GE has been recently proposed [4].

The aim of the present paper is a further elucidation of the conundrum of whether the rise of a valley succeeds or is flattened and disappears at the ends. In general, this happens in regions of the PES far away from stationary points (where bifurcations can emerge also [6]). Stable molecular vibrations need a convex bowl at the bottom of the PES, and a reaction goes over a SP. But before a chemical reaction starting in a bowl takes place the system first must be excited. It is possible that the reaction reaches very structured parts of the PES, in which case the valley may disappear somewhere in the mountains, the corresponding mode ceases to exist, its level ladder should end and its vibrational energy be redistributed into other modes. Hence, it is to be expected that changes in the PES valleys would be accompanied by strong changes of the spectroscopic behaviour of the molecule. It is therefore of great interest, to compute bifurcation points (BP) on a given PES and then to understand their influence on the spectrum of the molecule. The latter aim is the basis of a forthcoming paper by the author. The calculation of BPs is illustrated by some simple, but instructive, examples of analytic surfaces which give us the possibility of discussing the more abstract Basilevsky classification [7,8] of BP's with simple solvable equations. We conclude the mathematical treatment with a discussion of the "strange" third GE of the HNR example [1] in the light of the Basilevsky classification [8]. We show how only a small change in a parameter of the PES makes a qualitative change in the BP character.

In a physical discussion we compare the quasilinear behaviour and internal energy redistribution which are consequences of PES valley bifurcations. The molecule HCN should be an interesting candidate for understanding bifurcations of valley paths.

\section{Gradient extremals}

We recall the definition of Hoffman, Nord and Ruedenberg (HNR) [1] (slightly modified):

A gradient extremal (GE) intersects every contour line in that point where the absolute value of the gradient is an extremal value compared to other gradient values on the same contour.

(Gradients are always perpendicular to the contour line.) We introduce the so called defect functional [5] for the gradient norm

$$
\sigma(x, y)=\frac{1}{2}\|\operatorname{grad} U(x, y)\|^{2}=\frac{1}{2} U_{x}^{2}+\frac{1}{2} U_{y}^{2}
$$

of the gradient $\left(U_{x}, U_{y}\right)$ where $U(x, y)$ is the energy surface over a $2 \mathrm{D}$ configuration space $\boldsymbol{R}^{2}$. We use the standard partial derivative notation. The way to 
determine an extremal of the defect functional $\sigma$ along a contour line of $U(x, y)$ is to search for solutions of

$$
t \cdot \operatorname{grad} \sigma=0,
$$

where $t$ is the unit tangent vector of the contour line $U(x, y)=$ constant or $y=\mathrm{cl}(x)$, and $\operatorname{grad} \sigma=\boldsymbol{H} \cdot \operatorname{grad} U$. (We use small initial letters of a contour line $\mathrm{cl}(x)$ for an explicit functional relation and capitals for corresponding implicit definitions). Equation (2) can be understood as a condensation of a formula for the direction derivative of the $\sigma$-functional along $\mathrm{cl}(x)$. The vector part of grad $\sigma$ in directions other than $t$ is cancelled by the scalar product with $t$. Condition equation (2) remains in the assumed extremal condition along $\mathrm{cl}(x)$. In the 2D case we simply have

$$
t=\left(U_{y},-U_{x}\right) /(2 \sigma)^{1 / 2},
$$

and we get from Eq. (2) the equation for a gradient extremal [1]

$$
\mathrm{GE}(x, y)=U_{x y}\left(U_{x}^{2}-U_{y}^{2}\right)+\left(U_{y y}-U_{x x}\right) U_{x} U_{y}=0 .
$$

At first sight, Eq. (3) seems to be a differential equation [1], but here we have to differentiate the given energy $U=U(x, y)$ at any argument $(x, y)$. The result at a point $(x, y)$ is a value, zero or nonzero. The requirement for $\mathrm{GE}$ to be zero in Eq. (3) makes a (maybe implicit) functional relation of $x$ and $y$, not a differential equation. Any couple $(x, y)$ of the configuration plane can be tested in Eq. (3). In general only a subset of the plane will give a solution. But already in the 2D case it is not clear whether we get a 1D curve $y=$ ge $(x)$. We show this by a simple counterexample:

Example 1. The model energy is a paraboloid of rotation

$$
U(x, y)=x^{2}+y^{2}
$$

We get $U_{x y}=0, U_{x x}=2, U_{y y}=2$, and

$$
\mathrm{GE}(x, y)=0\left(4 x^{2}-4 y^{2}\right)+0(4 x y)=0 .
$$

Every point $(x, y)$ fulfils the GE equation. Thus, the whole plane is itself GE. At all points except the origin there are two eigenvector fields, one tangent to the profile parabola, the other tangent to the circle of revolution in a horizontal plane.

If we have a somewhat more complicated surface $U(x, y)$ we can assume that we get a $1 \mathrm{D}$ curve solving the GE condition equation (3). We emphasize that we look for curves $\mathrm{GE}(x, y)=0$ or $y=\operatorname{ge}(x)$ in the $(x, y)$-plane. The valley floor path on the potential landscape then is the energy profile over this GE curve or, conversely, the GE is the projection of the valley floor on $U(x, y)=U(x$, ge $(x))$ into the $(x, y)$-plane.

Properties of GE's are outlined [1]. (1) All stationary points are on GE because there $U_{x}=U_{y}=0$. (2) Further we have the somewhat surprising behaviour that, 
in general, the direction of the gradient and of the tangent to a GE form a finite angle.

To understand point (2) we look at $\operatorname{GE}(x, y)=0$ as an implicit definition of a curve $y=$ ge $(x)$, and we assume a displacement $(d x, d y)$ along the GE. We get from $\mathrm{Eq} .(3), \mathrm{GE}(x, y)=0$, that

$$
\mathrm{GE}_{x} d x+\mathrm{GE}_{y} d y=0,
$$

or

$$
\frac{d y}{d x}=\operatorname{ge}^{\prime}(x)=-\mathrm{GE}_{x} / \mathrm{GE}_{y}
$$

(if $\mathrm{GE}_{y} \neq 0$ ). It seems (without any proof here) easy to believe that the two vectors $\left(U_{x}, U_{y}\right)$ and $\left(-\mathrm{GE}_{y}, \mathrm{GE}_{x}\right)$ point in different directions because Eq. (4) has third order derivatives of $U$ of [1].

To discuss further details we look for some simple model surfaces. First we give a model containing three GE's with a very normal behaviour.

\section{Example 2.}

$$
U(x, y)=\frac{1}{2}(x y+2)(y-x) .
$$

We find three valleys and three ridges meeting in a central region. We denote valleys by $v_{1}, v_{2}, v_{3}$ and ridges by $r_{1}, r_{2}$ and $r_{3}$, clockwise. From Eq. (3) we find the formula

$$
\begin{aligned}
\mathrm{GE}(x, y)=(x+y)\left(2+x y-(y-x)\left((y-x)^{2}-2\right)^{1 / 2}\right) \\
\times\left(2+x y+(y-x)\left((y-x)^{2}-2\right)^{1 / 2}\right)=0,
\end{aligned}
$$

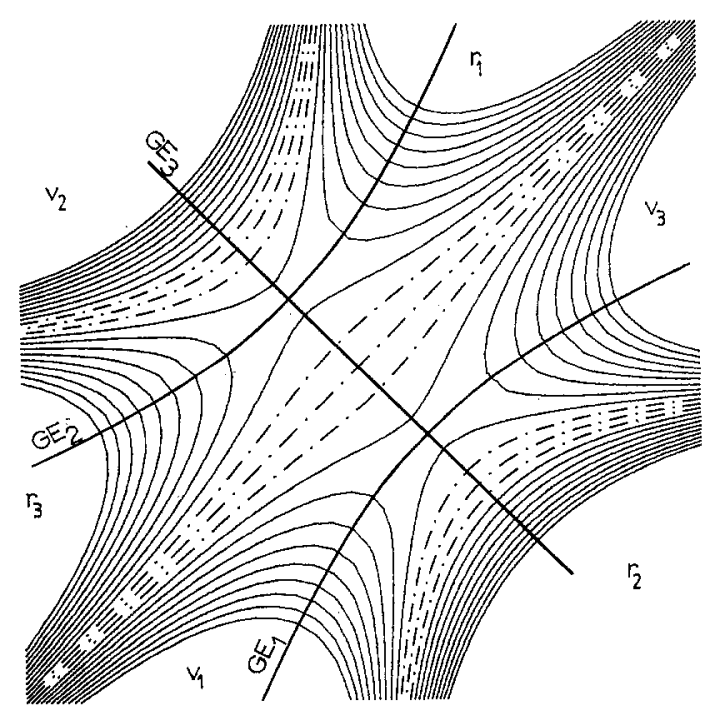

Fig. 1. Model surface $U(x, y)=$ $\frac{1}{2}(x y+2)(y-x)$, where $v_{i}$ is a valley, $r_{i}$ a ridge, GE a gradient extremal (fat curves). Contour lines, except for those at $-0.5,0$ and 0.5 (dotdashed), are solid 
where the three factors give three different branches of the solution of the GE condition. The valleys $v_{1}$ and $v_{3}$ are connected by a GE over $\mathrm{SP}_{2}$ numbered $\mathrm{GE}_{1}$, the ridges $r_{3}$ and $r_{1}$ are connected by GE No. 2 over $\mathrm{SP}_{1}$. (Of course, the GE definition equation (2) makes no difference between the floor line of a valley and the crest of a ridge. In both cases $\sigma$ is minimal.) Note that $\mathrm{SP}_{2}$ is lower than $\mathrm{SP}_{1}$. The third straight $\mathrm{GE}_{3} y=-x$ coming uphill out of $v_{2}$ meets the $\mathrm{SP}_{1}$, then goes downhill through point $(0,0)$ to $\mathrm{SP}_{2}$ and at last it goes again up the ridge $r_{2}$, see Fig. 2. In the HNR classification [1] the $\mathrm{GE}_{3}$ in $v_{2}$ traces a cirque and on $r_{2}$ it traces a cliff. The point $(0,0)$ is a so-called valley-ridge inflection point [6], but we choose to call it cirque-cliff inflection (CCI) point as this is consistent with [1], see Fig. 3. The three GE's of Fig. 1 meet exactly in the two SP's and no further bifurcation emerges. The CCI point is not a bifurcation point. Trajectories orthogonal to the contour lines are possible in the six sections of the plane divided by the GE's. Thus, except for $\mathrm{GE}_{3}$ itself, there exists no steepest descent line from any ridge to its opposite valley.

\section{Example 3.}

$$
U(x, y)=\frac{1}{2}(x y-2)(y-x) \text {. }
$$

Now, we find the SP's to be on the same contour line, $y=\operatorname{cl}(x)=x$ of height zero, as the CCI point. With a little imagination one can imagine Fig. 4 as a double "monkey" saddle where the one SP is flattened to two SP's and a CCI point in between, cf Fig. 5. One readily ascertains from Eq. (3) the condition

$$
\begin{aligned}
\mathrm{GE}(x, y)=(x+y) & \left(2-x y-(y-x)\left((y-x)^{2}+2\right)^{1 / 2}\right) \\
\times & \left(2-x y+(y-x)\left((y-x)^{2}+2\right)^{1 / 2}\right)=0
\end{aligned}
$$

which again gives three GE branches. $\mathrm{GE}_{3}$ from northwest to southeast goes steadily uphill through the CCI point $(0,0) . \mathrm{SP}_{1}$ connects $v_{1}$ and $v_{2}$, and $\mathrm{SP}_{2}$ connects $v_{3}$ and $v_{2}$. It seems that we find no connection between $v_{1}$ and $v_{3}$, or $r_{1}$ and $r_{3}$. Two branches of $\mathrm{GE}_{1}$ coming from $v_{1}$ and $v_{3}$ go over $\mathrm{SP}_{1}$ and $\mathrm{SP}_{2}$, as expected, and flow into valley $v_{2}$. There they meet at the point

$$
\mathrm{BP}_{1}=\left(-\left(\frac{2}{5}\right)^{1 / 2},\left(\frac{2}{5}\right)^{1 / 2}\right) \text {. }
$$

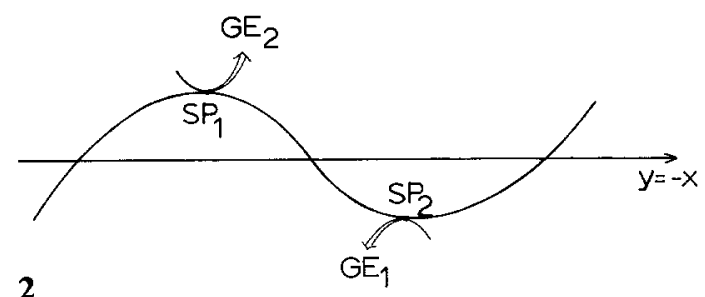

Fig. 2. Energy profile over $\mathrm{GE}_{3}$ from northwest to southeast in Fig. 1

Fig. 3. Illustration of contours (solid lines); a GE for a valley (dashed line); a GE for a ridge (dot-dashed line); a GE along cirque; (dotted) CCI point; and cliff from bowl B to summit S 


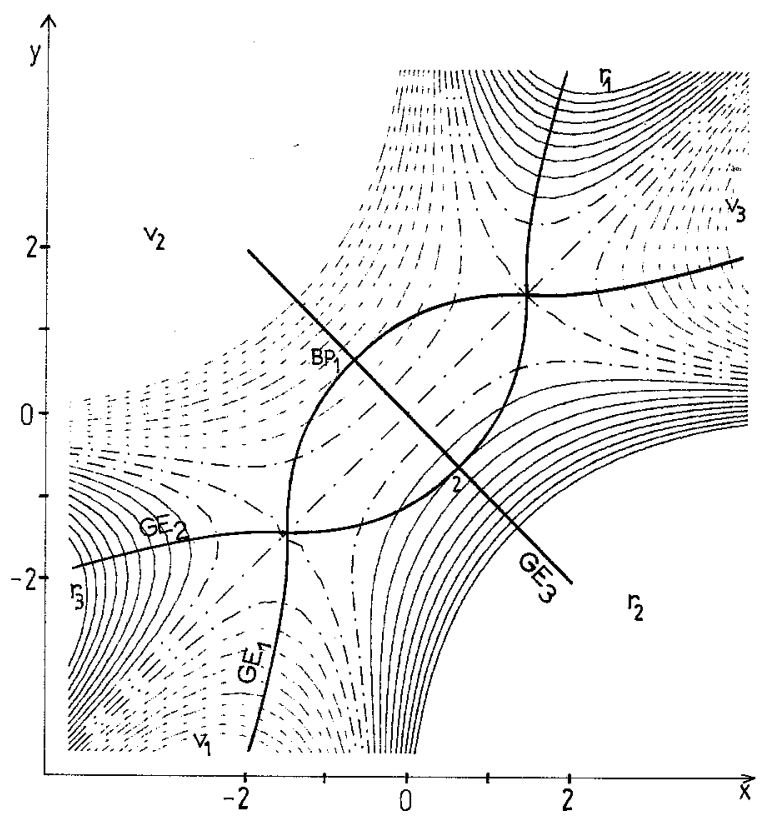

Fig. 4. Model surface $U(x, y)=$ $\frac{1}{2}(x y-2)(y-x)$, where BP is a bifurcation point

Analogously, two branches of $\mathrm{GE}_{2}$ going down ridges $r_{1}$ and $r_{3}$ cross the SP's and meet at

$$
\mathrm{BP}_{2}=\left(\left(\frac{2}{5}\right)^{1 / 2},-\left(\frac{2}{5}\right)^{1 / 2}\right)
$$

on the ridge $r_{2}$ crossing $\mathrm{GE}_{3}$. If we look at the valleys clearly we expect a bifurcation of $v_{2}$ into $v_{1}$ and $v_{3}$. The bifurcation point (BP) on the ascending $\mathrm{GE}_{3}$ indeed exists but the two side valleys emerge only very slightly. We get two

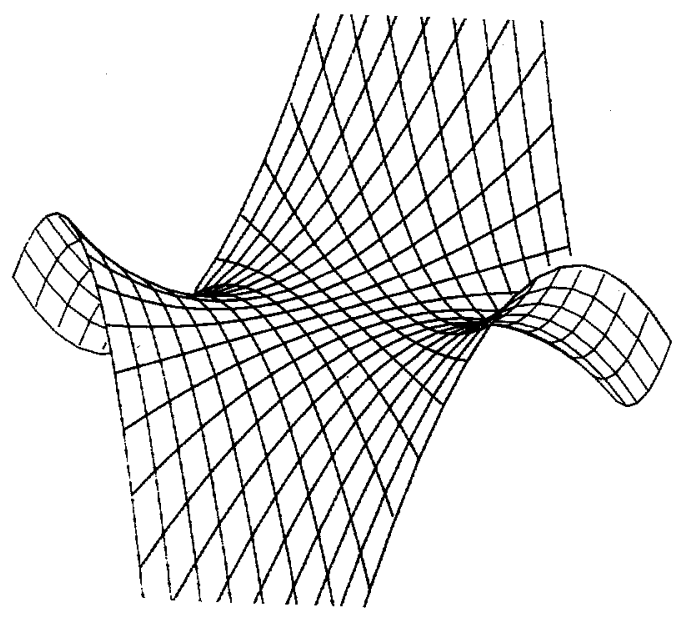

Fig. 5. View uphill on the surface of Fig. 4 from valley $v_{2}$ along $\mathrm{GE}_{3}$ 
BP's of $\mathrm{GE}_{3}$ by a formula system of Basilevsky [7]

$$
\begin{aligned}
u_{t t}\left(u_{t t}-u_{g g}\right)+u_{g} u_{g t t} & =0, \\
u_{t g} & =0,
\end{aligned}
$$

where $g, t$ denotes direction derivatives in the gradient and the tangential direction on the contour line. In the symmetric model of Eq. (6), if we are interested only in the points of the diagonal $\mathrm{GE}_{3}$ with $y=-x$, the direction derivatives can be easily obtained by a $\pi / 2$ rotation of the $(x, y)$ coordinates in $(g, t)$ "coordinates":

$$
x=(t-g) / 2^{1 / 2}, \quad y=(t+g) / 2^{1 / 2} .
$$

We get the representation of the surface equation (6)

$$
u(t, g)=g\left(t^{2}-g^{2}-4\right) / 2^{3 / 2}
$$

and the derivatives

$$
\begin{aligned}
& u_{g}=\left(t^{2}-3 g^{2}-4\right) / 2^{3 / 2}, \quad u_{t}=t g / 2^{1 / 2}, \\
& u_{g g}=-3 g / 2^{1 / 2}, \quad u_{t t}=g / 2^{1 / 2}, \quad u_{t g}=t / 2^{1 / 2}, \\
& u_{g t t}=1 / 2^{1 / 2} .
\end{aligned}
$$

With condition equation (7) we get $t=0,5 g^{2}+t^{2}-4=0$,

Thus,

$$
g= \pm \frac{2}{5} 1 / 2
$$

and by a back-transformation the given BP's in $(x, y)$ coordinates result. They are the triple points $\psi$ and $\phi$ in the Basilevsky classification [8].

What happens in a bifurcation point which is no SP? On $\mathrm{GE}_{3}$ the gradient takes a maximal value if we test it over a contour line, see Fig. $6 \mathrm{a}$. Thus, $v_{2}$ is a cirque throughout and $r_{2}$ a cliff [1]. In BP's, a $\sigma$-curve over a contour line flattens to a horizontal line, (Fig. 6b). In a local neighbourhood of the BP, every point of the

Fig. 6. $\sigma$ profile over contour lines (cl) of Fig. 4. a cl through point $(-1,1)$; $\mathrm{b}$ cl through $\mathrm{BP}_{1}$, c cl through point $(0,0)$

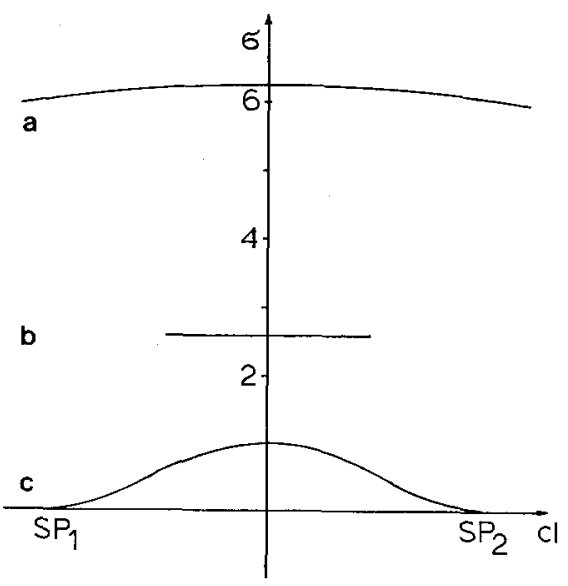


contour line shows the same $\sigma$ value. Locally we therefor have a situation analogous to Example 1, since we get the GE property for a whole piece of a contour line. Between $\mathrm{BP}_{1}$ and the $\mathrm{CCI}$ point $(0,0)$ in addition to the maximal $\sigma$ on the contour line indicating $\mathrm{GE}_{3}$, we get two minimal $\sigma$ points indicating the branches of $\mathrm{GE}_{1}$ (Fig. $6 \mathrm{c}$ ). On $\mathrm{GE}_{3}$, the ray $y=-x$, we have the values

$$
\sigma=\left(1+\frac{3}{2} x^{2}\right)^{2}
$$

and on the contour line zero we have $\sigma=\left(1-x^{2} / 2\right)^{2}$. Going out of $\mathrm{SP}_{1}$, for an orthogonal trajectory we observe the same direction as for $\mathrm{GE}_{1}$. Thus, $\mathrm{GE}_{1}$ indicates the real valley over $\mathrm{SP}_{1}$ here, and later $\mathrm{GE}_{1}$ turns to the right with its valley ending by flattening in $\mathrm{BP}_{1}$ on the slope of cirque $v_{2}$. The valley of $\mathrm{GE}_{1}$ near $\mathrm{BP}_{1}$ is so imperceptible that the gradient of the surface nearly suppresses its influence. Orthogonal trajectories flowing over the piece of the axis $y=x$ between the SP's (we see a dike) cross the $\mathrm{GE}_{1}$ in cirque $v_{2}$ and the $\mathrm{GE}_{2}$ on cliff $r_{2}$, almost without distortion. The reason is the possible divergence of the direction of gradient and of the vector tangential to a GE mentioned above.

Again in the CCI point we do not find any branching. In $(0,0)$ we have a nonzero gradient, but the Hessian

$$
\boldsymbol{H}=\left(\begin{array}{cc}
-y & y-x \\
y-x & x
\end{array}\right)
$$

also has two zero eigenvalues, as in Example 2. So, here a "branching" condition for a steepest descent path is fulfilled [7]:

$$
u_{g g}=0, \quad u_{t g}=0 .
$$

The CCI point lies on the straight contour line $y=x$ where we have the orthogonal gradient direction $(1,-1)$, except at the two SP's where the gradient vanishes. This is the direction of the $\mathrm{GE}_{3}$ itself. In Example 2 we find the analogous gradient $(-1,1)$. Hence, there is no branching of an orthogonal trajectory, and it follows that condition equation (8) (Eq. (7) of [7]) is not sufficient. Orthogonal trajectories cannot bifurcate on a surface which is continuous and smooth, except at stationary points, cf [6]. In minima they meet asymptotically from nearly all directions along the direction of the smallest eigenvalue.

Example 4. We treat a modified HNR model

$$
U(x, y)=\frac{1}{2}\left(x y^{2}-y x^{2}+\mu x^{2}+2 y-3\right),
$$

where we include a parameter $\mu$. Here, $\mu=1$ is the HNR surface [1]. It is easy to execute derivations of the GE condition equation (3) with this model surface equation (9). It gives a polynomial of fifth degree in $x$ and $y$. Even for $\mu=1$, it is not easy to factorize. Hence, the GE condition implicitly defines the GE curves. If we use the advantage of a $2 \mathrm{D}$ model, we can solve it by a point-by-point calculation over a close grid and draw contour curves, $\mathrm{GE}(x, y)=$ constant, including the zero curve. This is done in Figs. 7-9 (cf. Fig. 2 in [1]). Model 4 is an asymmetric distortion of models 2 and 3 . This results in a dramatic change 
Fig. 7. Model surface $U(x, y)=$ $\frac{1}{2}\left(x y(y-x)+1.15 x^{2}+2 y-3\right)$ including GE's, SP denotes a saddle point

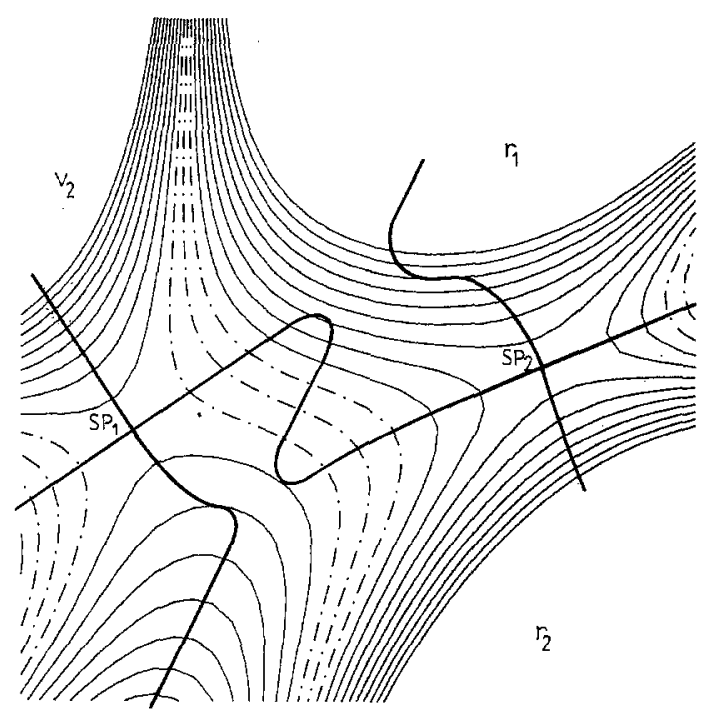

of the GE behaviour in the central region of the surface. With the experience of Examples 2 and 3 we can give an explanation of the "strange" central pieces of the GE's in Figs. 7-9. In Fig. 8 we observe a straight $G_{1}$ from south to north, i.e. from $v_{1}$ to $r_{1}$. The two other GE's cross $\mathrm{GE}_{1}$ at true BP's of the type in Example 3, but with a nonorthogonal crossing angle. If we vary the parameter $\mu$ we shift mainly the $\mathrm{SP}_{2}$. For $\mu>1.08$ it is raised, for $\mu<1.08$ it is lowered. In both cases we "lacerate" the BP's and only retain some turning points of the corresponding GE's. The turning point character of the central GE in the former

Fig. 8. Model surface $U(x, y)=$ $\frac{1}{2}\left(x y(y-x)+1.08 x^{2}+2 y-3\right)$ including GE's

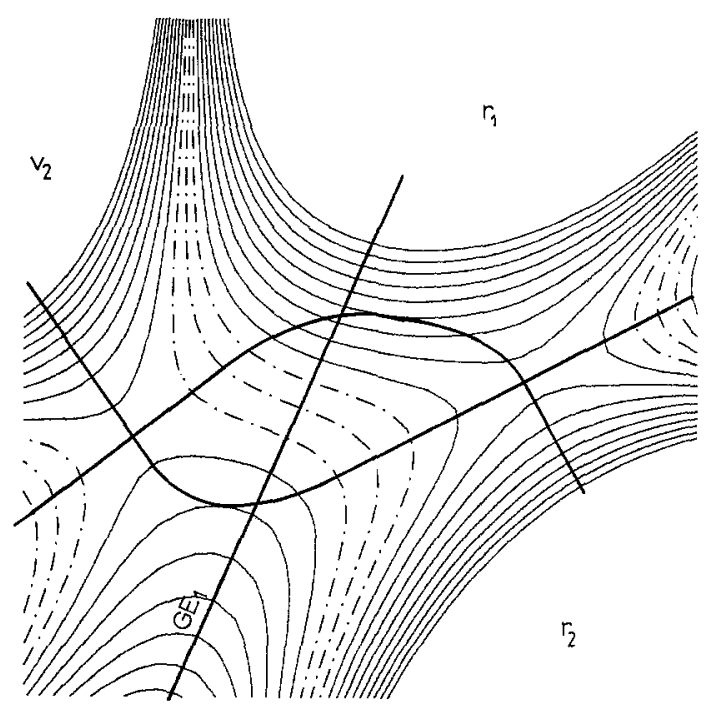


bifurcation region can be better observed in case $\mu=1.15$ (Fig. 7), because here branches which meet at an acute angle are connected. In the HNR case [1] with $\mu=1.0$ we get obtuse-angled branches.

With $\mathrm{GE}_{1}$ (valley floor path) and $\mathrm{GE}_{2}$ (ridge crest path) the representation in Fig. 9 gives a pattern as in Example 2. Only the $\mathrm{GE}_{3}$ seems very strange, showing a strong deviation from the straight path of Example 2 in the central region.

The mathematical question of how to calculate the $\mu$ value where a bifurcation exists is quite a difficult one. Eq. (7) cannot directly be used because we do not know the corresponding gradient and contour line tangent at this point. So we cannot calculate the direction derivatives in condition equation (7). For a more detailed discussion see [9].

Now, let us discuss the HNR case $\mu=1$ in some detail. In valley $v_{1}$ with $U(x, y)<-1.5$ we find normal behaviour; namely the existence of a unique $\mathrm{GE}_{1}$ tracing the valley floor and coming from minus infinity. $\mathrm{GE}_{1}$ then turns up to the right near the point $(0.4,-0.05)$ toward $\mathrm{SP}_{2}$. On contour lines lower than -1.5 in $v_{1}$, at the crossing with $\mathrm{GE}_{1}$, we have by definition an extremal of $\sigma$, namely a minimal value. Indeed, if we leave the $\mathrm{GE}_{1}$ crossing along a contour line to the right or to the left we get an increase of $\sigma$. At first sight, we can check this by a continuous shortening of the distance to the next contour line. But on the contour line $(-1.5)$ walking through $(0,0)$ the $\sigma$ profile suffers a change: a shoulder emerges. We have $U_{x}(0,0)=0, U_{y}=\frac{3}{2}$, and the Hessian elements

$$
U_{x x}=1, \quad U_{y y}=0, \quad U_{x y}=U_{y x}=0 .
$$

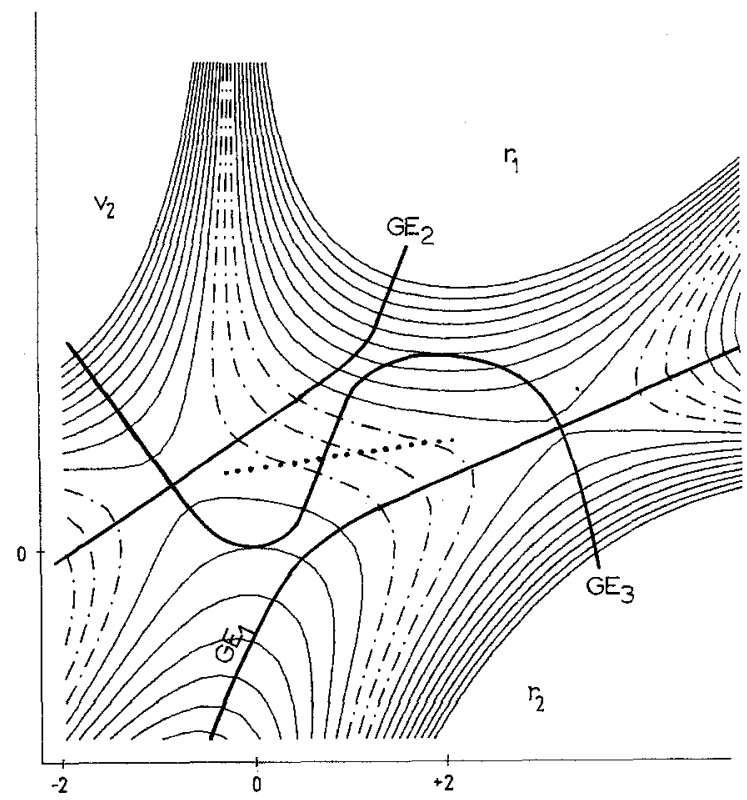

Fig. 9. Model surface $U(x, y)=$ $\frac{1}{2}\left(x y(y-x)+x^{2}+2 y-3\right)$, cf [1], including GE's and a piece of the contact line (dotted) 
The gradient actually pointing in the $y$-direction is parallel to an eigenvector (which in addition is a zero eigenvector). Hence, the point $(0,0)$ is a GE point. It is a double point in the Basilevsky classification [8]. If we go uphill to the left from $(0,0)$ we can now trace a new valley floor of $\mathrm{GE}_{3}$ up to $\mathrm{SP}_{1}$ which must be characterized by a second minimum of $\sigma$. The $\sigma$ shoulder of the $\sigma$ profile over $(0,0)$ on the $(-1.5)$-contour line splits on higher contour lines into a minimum and a maximum. In Fig. 10 the $\sigma$ profile along the contour line $(-1)$ is given. Clearly, between the two minima there exists a maximum, but in contrast to Example 3 and to the case $\mu=1.08$, the origin of the two new $\sigma$ extremals is outside the old $\mathrm{GE}_{1}$. The maximal $\sigma$ value belongs to the central arc of $\mathrm{GE}_{3}$ between $(0,0)$ and $(2,2)$. It begins at $(0,0)$ in tracing the end cirque of $v_{1}$. Going uphill it does not find a simple CCI point but a broad intermediate region which is characterized by contact of a flank of the central ridge $r_{1}$ and of a flank of $v_{1}$ leading to $\mathrm{SP}_{2}$. We can define a contact line of the two flanks by connecting those points on contour lines having curvature zero. If we walk along a contour line (cl) we go from convex behaviour in valley $v_{1}$ to concave behaviour on ridge $r_{1}$. Thus, the equipotential line somewhere shows a change of curvature from plus to minus: this is the point on the contact line. We again treat $U(x, y)=$ constant as an implicit definition of the contour line $y=\mathrm{cl}(x)$ and get the condition

$$
\mathrm{cl}^{\prime \prime}(x)=\left(2 U_{x} U_{y} U_{x y}-U_{y}^{2} U_{x x}-U_{x}^{2} U_{y y}\right) / U_{y}^{3}=0,
$$

(if $\left.U_{y} \neq 0\right)$. We set $\tilde{\boldsymbol{t}}=\left(U_{y},-U_{x}\right)$, which is a non normed tangential vector orthogonal to the gradient of $U$. Eq. (11) gives the condition

$$
\tilde{\boldsymbol{t}} \cdot \boldsymbol{H} \cdot \tilde{\boldsymbol{t}}=\mathbf{0}
$$

for a point $(x, y)$ to be an inflection point on a contour line where two flanks stick together. In general, the $\mathrm{GE}_{3}$ passing the $\mathrm{CCI}$ belt and the contact line dividing the flanks of ridge $r_{1}$ and valley $v_{1}$ do not coincide. They do, however, cross at the point $(0.685,0.920)$, cf [1], where the Hessian $\boldsymbol{H}$ has a zero eigenvector parallel to $\tilde{t}$. At this point for Eq. (3) we get

$$
(\boldsymbol{t} \cdot \boldsymbol{H}) \cdot \boldsymbol{g}=\mathbf{0} \cdot \boldsymbol{g}=0 .
$$

Thus, it is on the $\mathrm{GE}_{3}$. On the rest of the contact line we still have a zero eigenvalue of $\boldsymbol{H}$, but the corresponding eigenvector is not parallel to $\boldsymbol{t}$, (a behaviour

Fig. 10. $\sigma$ profile over the contour line $(-1)$ in Fig. 9

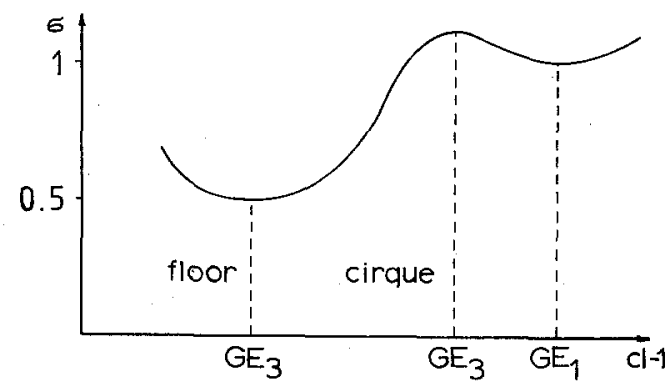


which is also seen near the SP's). From point $(0.685,0.920)$ downhill to $(0,0)$ the $\mathrm{GE}_{3}$ traces the maximal cirque region of the flank of valley $v_{1}$, and uphill to $(2,2)$ the $\mathrm{GE}_{3}$ traces the maximal cliff region of the flank of $r_{1}$. So, over three contour lines the CCI belt substitutes for the CCI point as in Examples 2 and 3. The model surface in Fig. 9 consists of three valleys and three ridges meeting in an asymmetric central region. An intuitive concept of a valley $v_{1}$ bifurcating uphill to $\mathrm{SP}_{1}$ and $\mathrm{SP}_{2}$, and of a ridge $r_{1}$ also bifurcating downhill to $\mathrm{SP}_{1}$ and $\mathrm{SP}_{2}$ is not supported by the GE result. The valley floor from $\mathrm{SP}_{1}$ downhill ends in $(0,0)$, analogously the crest to the right from $\mathrm{SP}_{2}$ uphill on $r_{1}$ in northwest direction ends in $(2,2)$ (cf an analogous result on another simple model surface in [10]). The end of a valley floor downhill is the mathematical result of a lacerated bifurcation point and of a new connected arc to a cirque ascending GE branch. It is characterized by Basilevsky's double point [8] which in mathematical terms is a turning point [9].

Of course, from any point of the final floor we can draw a steepest descent line and thus get a connection, or a bridge, over the region behind $(0,0)$, i.e. over the lacerated bifurcation region. The numerical result of a GE test in this region gives $\mathrm{GE}(x, y) \neq 0$, but the deviation from zero is only small. We call such a bridged region a quasivalley from inspection of the 2D, Fig. 9. The former bifurcation point of GE's in Fig. 8 for the case $\mu=1.08$ now changes in a flat SP region of a GE surface surrounded by zero contour lines. These zero equipotential lines are the GE's of the model surface.

\section{Discussion}

On PES's we do not generally find a coincidence of SP's CCI points and BP's. With clear definitions of these points on the GE curves, we now have a well suited instrument for characterizing PES's.

First of all, a first order SP gives the globally minimal energy required to drive a reaction of a chemical configuration over the corresponding transition structure. It should be noted that SP's of higher order are also of some chemical interest [11]. From a first order SP we can trace a steepest descent trajectory starting in an eigenvector direction of the Hessian. This gives Fukui's IRC, the intrinsic reaction coordinate [12]. Using the terms of a very old paper this path is the watercourse [13], because the actual acceleration of a water drop is tangential to the negative gradient. In the general case, however, we have no local criterion for a steepest descent trajectory being the IRC $[2,3]$; i.e. there is no way to tell if a point is or is not on this IRC path by looking at the PES only in the neighbourhood of a point.

This deficiency is surmounted by the GE definition if we additionally test whether we get a valley or a ridge for a GE [1]. The price is more computation for both the Hessian elements and the curve tracing of a corresponding GE from any stationary point to another [4]. In general, the latter is complicated [9] and only theoretically possible. If we use crude curve tracing algorithms then sometimes we may hop over bifurcation regions and bridge different branches of GE's with 
diagonal turning points. In summary GE's are a tool to reach an SP on a 1D path in an "unknown" landscape.

The steepest descent path and GE are curves defined on the static PES: they are only a frame for the real dynamics of a molecular transition in a chemical reaction. We have seen in Example 3 how imperceptibly a side valley can emerge. Thus, a molecular vibration, a mode, will not feel a BP but will be stable in the region around the BP of a GE, as long as the contour lines are still convex. The situation changes dramatically behind the CCI point. Then the contour lines become concave, from the point of view of the mode, and the molecular vibration bouncing on the cliff shows a propensity to vibrational redistribution. We assume two possible kinds of qualitative behaviour.

(i) If we have a flat cliff which quickly goes over into a ridge and which is accompanied by two closely neighbouring valleys, then the mode bounces on a double minimum section of the PES. Such a vibration is quantum mechanically possible and the spectroscopic category of quasilinear molecules gives an example, cf [14] for a review. The angle of a corresponding valley floor with the vibration direction should not be greater than $25^{\circ}-35^{\circ}$ in the molecular geometry [14], Table XIII. Figure 13 in [14] is the commonly used representation of the quasilinearity problem. It shows different $1 \mathrm{D}$ potential energy curves of double minimum shape for different quasilinear states of the same mode, i.e. in a 2D view a ridge and two ascenting neighbouring valleys. The slightly strange character of a quasilinear mode is due to the fact that a stretch mode goes along the crest of a ridge.

(ii) If we have a broad and precipitous cliff then the mode cannot be stabilized and suffers a vibrational redistribution. Recently, we have assumed this to be the case in HCN [15] since even in its fundamental transition the $\nu_{1}$ mode, which is mainly the $\mathrm{CN}$ stretch, reaches a concave contour line in its compression phase. As a result, the quantum state (100) of HCN exist, but is expected to be quite unstable. We could assign the redistribution process to two effects, (i) the anomalous infrared excitation of single rotational lines of $\nu_{1}$ itself connected with a sudden redistribution to $3 \nu_{2}^{1}$ [15], and (ii) the known HCN gas laser [16].

Hence, CCI points or belts are very important for real molecular vibrations. Any CCI point, lies on a GE and if we trace a GE to reach an SP we also get the CCI points $[1,6]$. If the region around the CCI point is asymmetric it will be a factor which influences the direction of an excited vibration, i.e. a factor in the selection of possible reactions as we can imagine from Figs. 7-9.

Cautionary remark. The definition of a valley floor, and in general of a GE, is a metric dependent concept and one needs to consider explicitly what is the physically relevant metric in every instance, of the discussion in $[2,3]$. This is because of the metric dependence of the Hessian matrix [17]. An invariant formulation of GE's is given in $[18,19]$, cf also the textbook [20]. 


\section{References}

1. Hoffman DK, Nord RS, Ruedenberg K (1986) Theor Chim Acta 69:265-279

2. Quapp W, Heidrich D (1984) Theor Chim Acta 66:245-260

3. Quapp W, Dachsel H, Heidrich D (1988) Submitted for publication in Z Phys Chem (Leipzig)

4. Jørgensen P, Jensen HJA, Helgaker T (1988) Theor Chim Acta 73:55-65

5. McIver JW, Komornicki A (1972) J Am Chem Soc 94:2625-2633

6. Valtazano P, Ruedenberg K (1986) Theor Chim Acta 69:281-307

7. Basilevsky MV (1987) Theor Chim Acta 72:63-67

8. Basilevsky MV (1982) Chem Phys 67:337-346

9. Mittelmann HD, Weber H (1980) Bifurcation problems and their numerical solution. Int Ser Num Math, vol 54, Birkhäuser, Basel

10. Rowe DJ (1982) Nucl Phys A391:307-326

11. Heidrich D, Quapp W (1986) Theor Chim Acta 70:89-98

12. Fukui K (1970) J Phys Chem 74:4161-4163

13. Maxwell JC (1870) Phil Mag 40:421, (1890) The Scientific Papers, vol II:233-240

14. Winnewisser BP (1985) In: Molecular spectroscopy: modern research, vol III. Academic Press, New York

15. Quapp W, Heidrich D (1988) Infrared Phys 28:83-89

16. Quapp W (1988) Appl Phys B in print

17. Tachibana A (1980) Theor Chim Acta 58:301-308

18. Basilevsky MV, Shamov SG (1981) Chem Phys 60:347-358

19. Rowe DJ, Ryman A (1982) J Math Phys 23:732-735

20. Gallot S, Hulin D, Lafontaine J (1987) Riemannian geometry (Universitext). Springer, Berlin Heidelberg New York Tokyo 